\title{
An Atypical Manifestation of Mycobacterium tuberculosis Infection
}

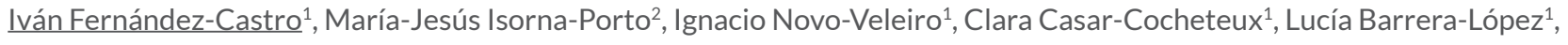 \\ Ariadna-Helena Andrade-Piña ${ }^{1}$, María López-Rodríguez ${ }^{1}$, Antonio Pose-Reino ${ }^{1}$ \\ ${ }^{1}$ Internal Medicine Service, Complexo Hospitalario Universitario, Santiago de Compostela, Spain \\ ${ }^{2}$ Internal Medicine Service, Hospital do Barbanza, Ribeira, Spain
}

Received: 07/05/2021

Accepted: 30/05/2021

Published: 05/07/2021

\begin{abstract}
How to cite this article: Fernández-Castro I, Isorna-Porto MJ, Novo-Veleiro I, Casar-Cocheteux C, Barrera-López L, Andrade-Piña AH, López-Rodríguez M, Pose-Reino A. An atypical manifestation of Mycobacterium tubercolosis infection. EJCRIM 2021;8: doi:10.12890/2021_002598.
\end{abstract}

Conflicts of Interests: The authors declare there are no competing interests.

This article is licensed under a Commons Attribution Non-Commercial 4.0 License

\section{ABSTRACT}

Introduction: Aortitis is seen in a wide variety of diseases. It was rarely found in the past but this is changing because of new imaging techniques. Case description: We present the case of a 45-year-old man who was found on thyroid ultrasound to have infrarenal aortitis and pathological lymphadenopathies in different locations. After an exhaustive diagnostic process, tuberculous aortitis, an infrequent manifestation of extrapulmonary tuberculosis, was diagnosed. The condition resolved after a 6-month course of antibiotics and a 6-week course of corticosteroids.

Conclusion: Tuberculous aortitis is an atypical manifestation of Mycobacterium tuberculosis infection. The absence of typical symptoms and the difficulty of isolating the microorganism makes its diagnosis difficult. Therefore, clinical suspicion, microbiological tests and imaging are key for reaching the diagnosis and starting treatment for a serious disease that can cause aortic aneurysm and dissection.

\section{LEARNING POINTS}

- New imaging techniques can identify aortitis for the diagnosis of extrapulmonary Mycobacterium tuberculosis infection.

- The extrapulmonary manifestations of Mycobacterium tuberculosis infection are diverse and include aortitis.

- Prompt and accurate differentiation between infectious and non-infectious causes of aortitis determines which of two very different therapeutic paths should be followed and hence the prognosis of the patient.

\section{KEYWORDS}

Aortitis, Mycobacterium tuberculosis

\section{CASE DESCRIPTION}

The authors describe the case of a 45-year-old man with history of obesity, dyslipidaemia, subclinical hypothyroidism, obstructive sleep apnoea and idiopathic generalized epilepsy. He was chronically medicated with valproic acid, levetiracetam, bromazepam, clobazam and a food supplement based on red yeast rice.

The patient was being followed-up by the endocrinology department for his obesity and thyroid disease. An ultrasound study to investigate his hypothyroidism incidentally showed multiple significant lymph nodes in the supraclavicular region. Computerized tomography (CT) confirmed these findings and also demonstrated bilateral mediastinal lymph nodes and abdominal aortitis (Figs. 1 and 2).

In the anamnesis the patient did not refer weight loss, anorexia or sweating, or to respiratory, digestive or cardiovascular symptoms. There was no report of recent contact with animals, travel abroad or unprotected intercourse. The patient also denied oral or genital ulcers, joint pain and Raynaud's disease. 


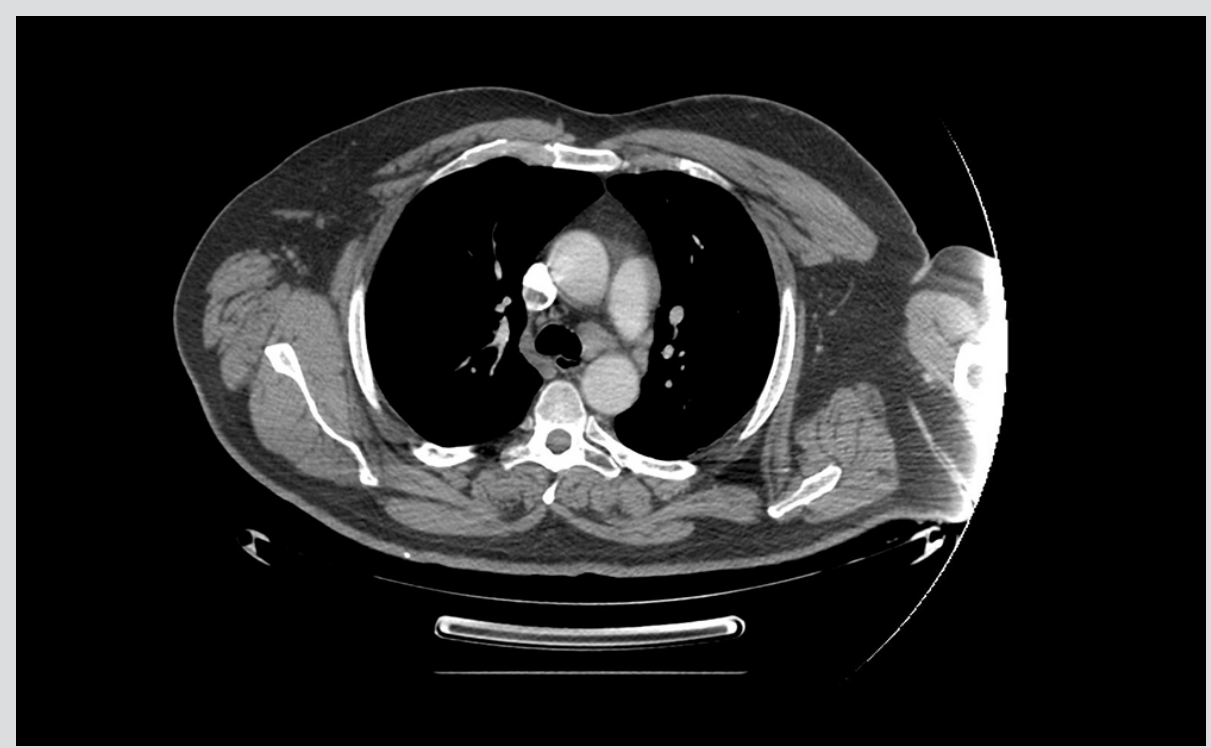

Figure 1. CT image showing mediastinal lymph nodes

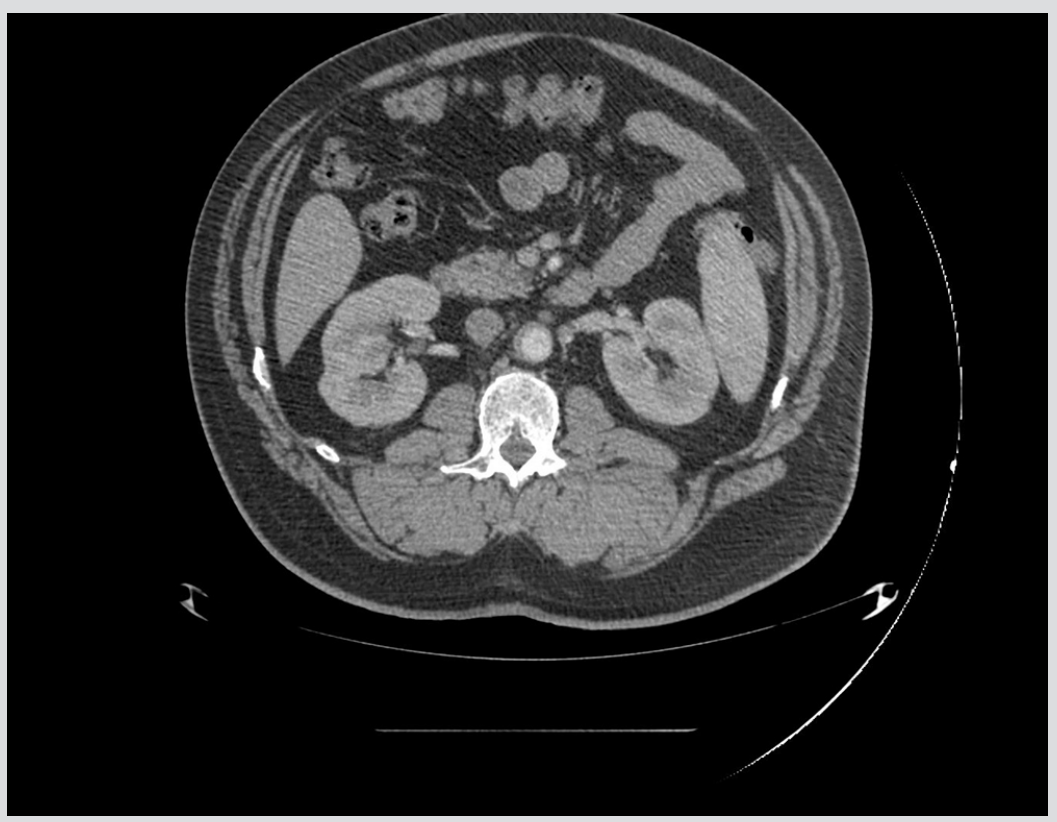

Figure 2. CT image showing abdominal aortitis

Cardiopulmonary auscultation was normal, and the abdomen was soft, without masses and not painful. Further investigation revealed a 1.5 $\mathrm{cm}$ supraclavicular lymph node, which was not painful, was elastic in consistency and was not fixed to an underlying structure.

A blood test showed no new alterations. In addition, we requested serological tests for HIV, Treponema pallidum, Toxoplasma gondii, EpsteinBarr virus (EBV), cytomegalovirus and viral hepatitis. Results demonstrated immunoglobulin G positive for EBV and T. gondii.

Positron emission tomography-computerized tomography (PET-CT) was performed and in addition to the lymphadenopathies described above, also showed significant uptake in subcarinal and paratracheal lymph nodes and in the left pulmonary hilum. The inflammation of the aortic wall seemed limited to the area between the exit of the renal arteries and the iliac bifurcation (Figs. 3 and 4). These findings suggested granulomatous inflammation but did not rule out a carcinogenic origin.

Thus, we were facing a case of aortitis with significant lymph nodes without a clear cause. At this point we decided to take a biopsy of one of lymph node, which showed granulomatous necrotizing lymphadenitis. The result of the biopsy was key to solving the case. The patient had aortitis and lymphadenopathy without apparent symptoms, implying a long-term process, and granulomatous necrotizing lymphadenitis. These findings suggested a slow-growing microorganism such as M. tuberculosis.

An anamnesis focused in this hypothesis revealed that in his childhood, our patient had a close contact with tuberculosis for which he took prophylactic treatment for 4 months. The diagnosis was confirmed after a positive tuberculin skin test, a positive interferon-gamma release 


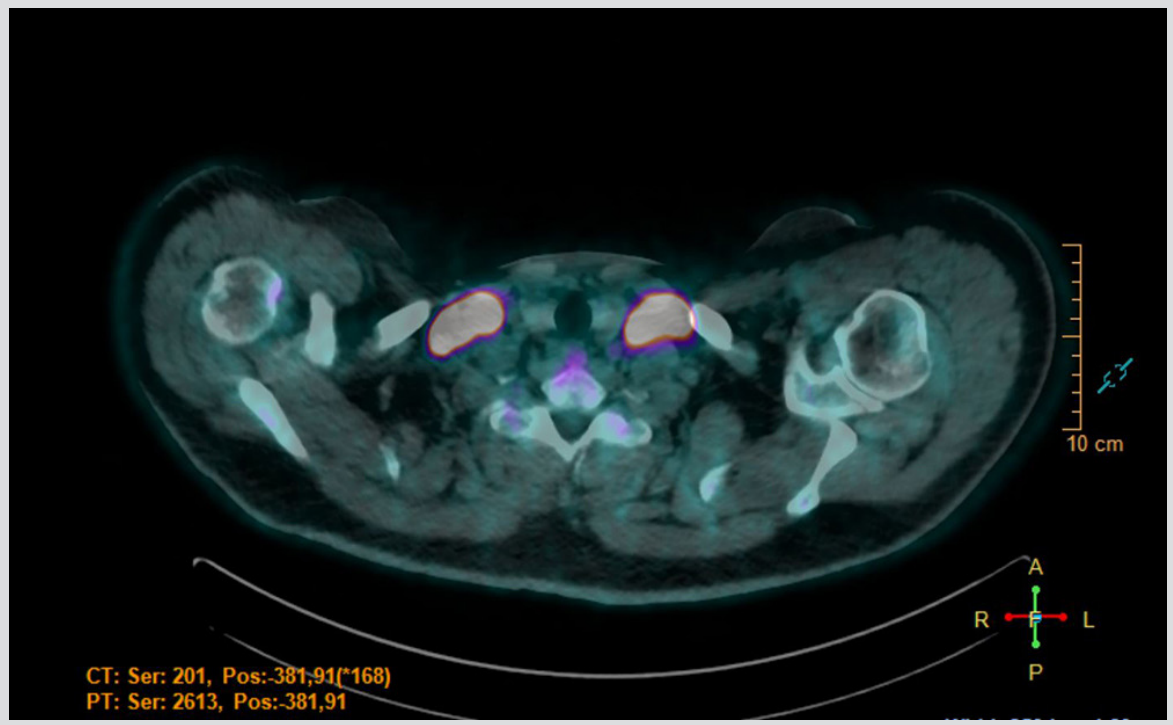

Figure 3. PET-CT image showing pathological uptake in the supraclavicular lymph nodes

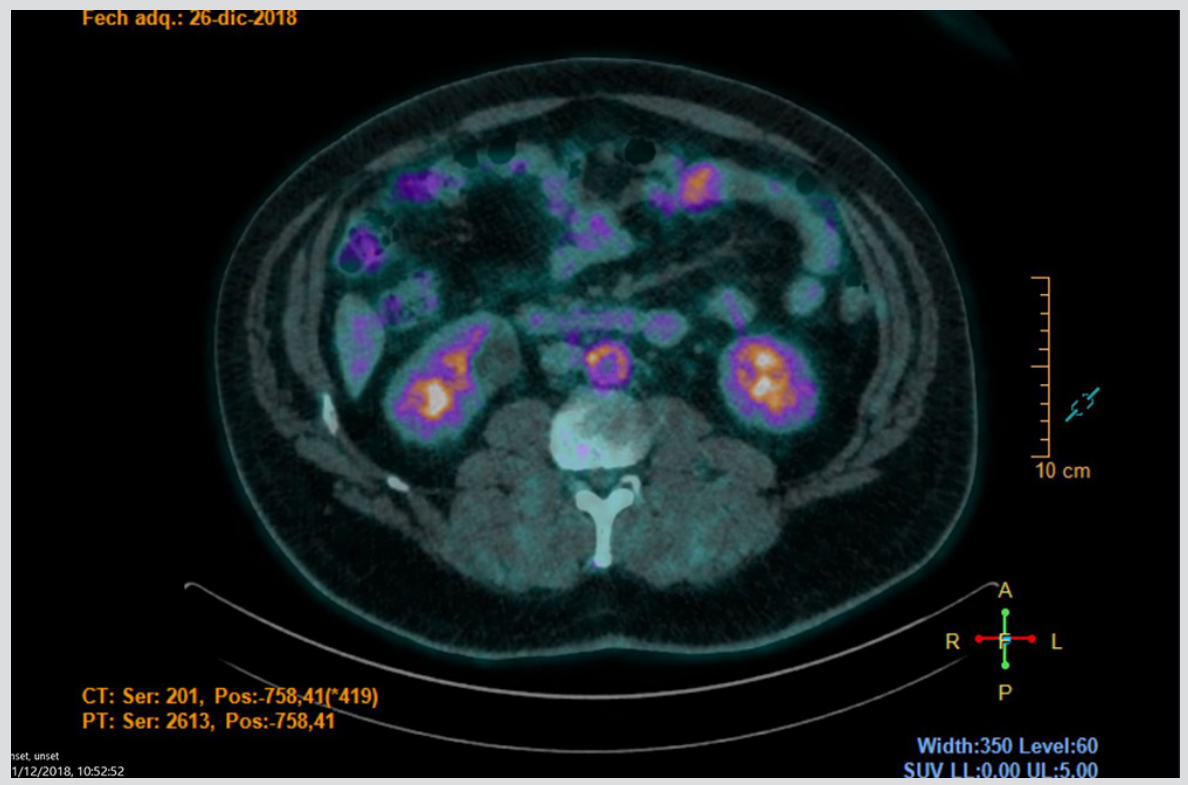

Figure 4. PET-CT image showing uptake in the abdominal aortic wall

assay (IGRA) and M. tuberculosis-positive culture of the lymph node biopsy sample. It should be noted that polymerase chain reaction (PCR) results were negative at first for $M$. tuberculosis.

Our patient was treated with anti-tuberculosis drugs for 6 mouths, with the addition of steroids during the first 6 weeks. PET-CT performed 1 and 2 years after the start of treatment showed that inflammation decreased and finally resolved.

\section{DISCUSSION}

Aortitis is an unusual manifestation of various diseases, and can have an infectious or non-infectious origin, so early distinction is essential for correct treatment ${ }^{[1]}$

Autoimmune diseases, especially giant cell and Takayasu arteritis, are the main causes of non-infectious aortitis. The clinical context, history and investigation results are key for correctly classifying the disease type ${ }^{[1]}$.

The incidence of infectious aortitis has been decreasing due to antibiotic treatment. Any microorganism can infect aorta. In the past, Treponema pallidum was the most frequent causative agent, but nowadays Gram-positive bacteria cause $60 \%$ of cases. Nevertheless, less frequent causes such as Gram-negative bacilli, M. tuberculosis, fungi or T. pallidum must also be kept in mind [2]. 
There are no specific biomarkers or imaging tests to conclusively identify aortitis, but some, like PET-TC, can help guide the differential diagnosis, response to treatment and need for surgery ${ }^{[1]}$. Uptake of fluorine-18-fluorodeoxyglucose indicates inflammation in the vascular wall, which should guide appropriate treatment. Lack of uptake indicates poor evolution and possible complications such as aneurysmal disease ${ }^{[3]}$.

M. tuberculosis is an unusual cause of aortitis. In one case series it was only found in $3.5 \%$ of patients with aortitis ${ }^{[4]}$, but we must take into account the significant population in contact with this microorganism.

The diagnosis of tuberculous aortitis is a challenge because the associated symptoms are unspecific ${ }^{[4]}$. Moreover, in cases of extrapulmonary tuberculosis, it can be even more difficult to detect the microorganism. In our case, we obtained a positive culture for the mycobacterium from the lymph node but such cultures can take 4-8 weeks to grow. Regarding the negative PCR, we must keep in mind that the sensitivity and specificity of this test are $85 \%$ and $92.5 \%$, respectively. In extrapulmonary tuberculosis, specifically with lymph node involvement, a combination of clinical suspicion, pathological anatomy, PCR, cytology and culture results will help reach the diagnosis.

Treatment is based on a regular antibiotic regimen for 6 months. There is no consensus on the use of corticosteroids, in contrast to other forms of extrapulmonary tuberculosis where they are clearly recommended. However, a series of 11 patients with tuberculous aortitis showed a good prognosis when treated with corticosteroid $0.25-2 \mathrm{mg} / \mathrm{kg} / \mathrm{day}$ for 6 weeks ${ }^{[4]}$.

Following these indications, our patient was treated with antibiotics for 6 months and prednisone 60 mg/day for 7 days with subsequent tapering with a favourable result (Fig. 5).

Aortic dissection, saccular aneurysm, pseudoaneurysm and stenosis are other possible and serious complications of this infection, in which case early surgery is essential to reduce mortality ${ }^{[4,5]}$.

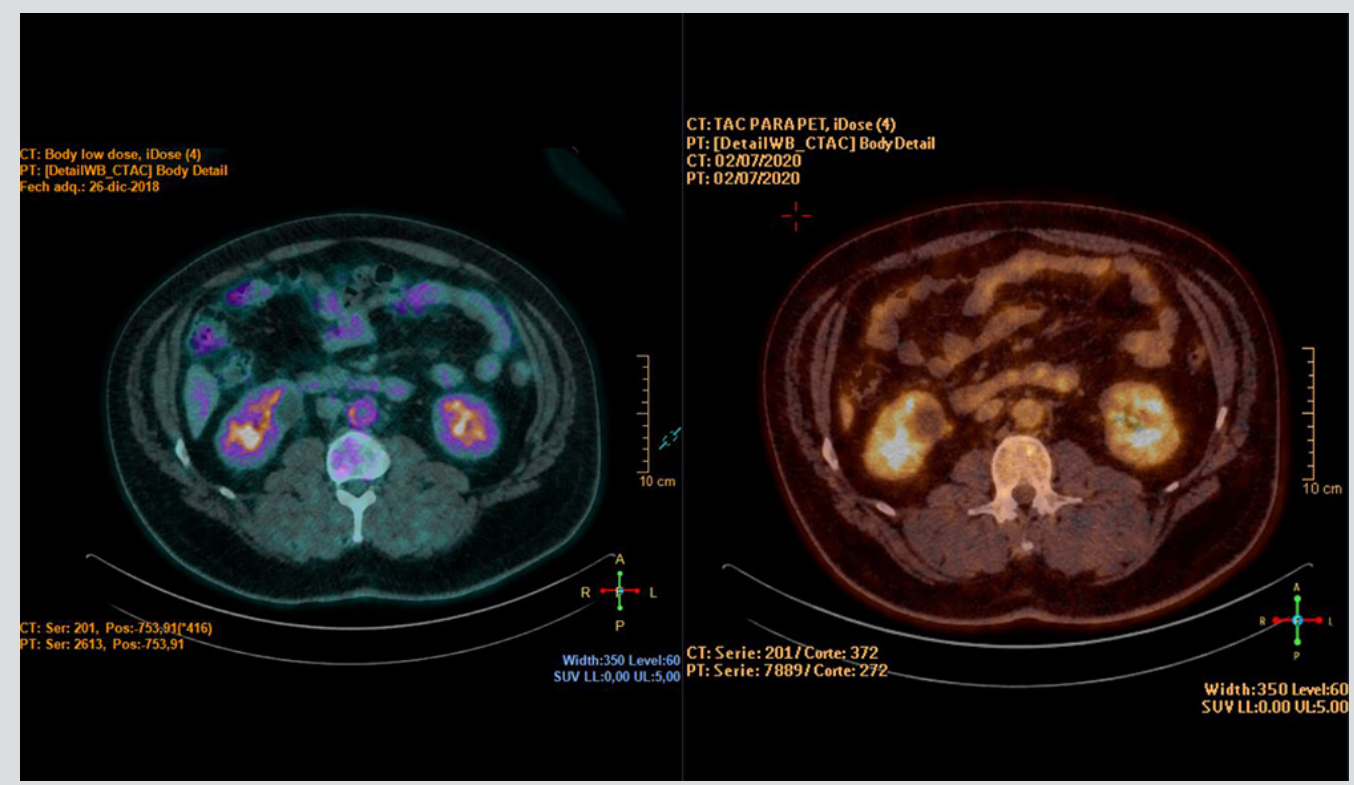

\section{REFERENCES}

Bossone E, Pluchinotta FR, Andreas M, Blanc P, Citro R, Limongelli G, et al. Aortitis. Vascul Pharmacol 2016;80:1-10.

Lopes RJ, Almeida J, Dias PJ, Pinho P, Maciel MJ. Infectious thoracic aortitis: a literature review. Clin Cardiol 2009;32(9):488-490.

Treglia G, Stefanelli A, Mattoli MV, Leccisotti L, Muoio B, Bertagna F. Usefulness of 18F-FDG PET/CT in evaluating disease activity at different times in a patient with chronic periaortitis. Nucl Med Mol Imaging 2013;47(1):69-71.

4. Delaval L, Goulenok T, Achouh P, Saadoun D, Gaudric J, Pellenc Q, et al. New insights on tuberculous aortitis. J Vasc Surg 2017;66(1):209-215.

5. Allins AD, Wagner WH, Cossman DV, Gold RN, Hiatt JR. Tuberculous infection of the descending thoracic and abdominal aorta: case report and literature review. Ann Vasc Surg 1999;13(4):439-444. 